Objectives/Aims Given the susceptibility to severe cutaneous viral infection and malignancy, we hypothesised there was a substantive defect in NK cell function in patients with DOCK8 deficiency.

Methods 10 patients with genetically confirmed DOCK8 deficiency as well as NK cell lines with stably reduced DOCK8 expression were evaluated experimentally using in vitro NK cell cytotoxicity, F-actin content, and confocal immunofluorescence microscopy assays.

Results DOCK8-deficient patients and cell lines all had decreased NK cell cytotoxicity and function could not be restored after IL-2 stimulation. Importantly, DOCK8 deficiency did not affect NK cell F-actin content, but impaired F-actin accumulation at the lytic immunological synapse.

Conclusions DOCK8 deficiency results in severely deficient NK cell function owing to an inability to form a mature lytic immunological synapse via focal F-actin accumulation. This defect may underlie important and previously perplexing attributes of the DOCK8 deficiency clinical syndrome including the unusual susceptibility to viral infection.

\section{A2.24 IL23/TH17-MEDIATED REGULATION OF ANTIBODY GLYCOSYLATION CONTROLS AUTOIMMUNE-INDUCED ARTHRITIS}

doi:10.1136/annrheumdis-2013-203215.24

\begin{abstract}
${ }^{1,2}$ René Pfeifle, 'Iryna Magorivska, ${ }^{4}$ Ulrich Scherer, ${ }^{~}$ Ulrike Harre, ${ }^{1,2}$ Tobias Rothe, ${ }^{3}$ Sybille Böhm, ' Martin Herrmann, ${ }^{5}$ Stephan Blüml, ${ }^{3}$ Falk Nimmerjahn, ${ }^{1}$ Georg Schett, ${ }^{1}, 2$ Gerhard Krönke. 'Department of Internal Medicine 3 and Institute of Immunology, University Erlangen Nuernberg; ${ }^{2}$ Nikolaus-Fibienger Center of Molecular Medicine, University Hospital and University of Erlangen Nuernberg; ${ }^{3}$ Department of Biology, University of Erlangen-Nuernberg; ${ }^{4}$ Department of Rheumatology Leiden, University Medical Center; ${ }^{5}$ University Hospital, Department of Internal Medicine 3 for Rheumatology, University of Vienna
\end{abstract}

Background and Objectives Both rheumatoid arthritis (RA) and the murine models of collagen-induced arthritis (CIA) and of $\mathrm{K} / \mathrm{BxN}$ arthritis are characterised by an initial break in self-tolerance, the appearance of specific autoantibodies and an autoantibody-mediated effector phase resulting in chronic inflammation and joint destruction. The IL23-dependent Th17 T-cell response has been identified as a major driving force during the pathogenesis of these disorders. The exact contribution of the IL23/Th17 axis to autoimmune-triggered inflammation, however, has remained incompletely understood. In this study, we aimed to further elucidate the role of IL23 and Th17 T-cells during murine autoimmune arthritis.

Materials and Methods To study and dissect the contribution of Th17 $\mathrm{T}$ cells to the initiation and effector phase of autoimmune arthritis, we performed the CIA as well as the $\mathrm{K} / \mathrm{BxN}$ serum transfer model of arthritis in both wild-type (WT) mice and in mice lacking the IL23-specific subunit $\mathrm{p} 19$. Subsequently we determined the clinical course of disease as well as the serum levels, the avidity and the glycosylation pattern of antibodies in the sera of the respective mice.

Results While IL23 $\%$ mice, which lack functional Th17 T-cells, developed a full-blown arthritis after passive transfer of autoantibodies in the $\mathrm{K} / \mathrm{BxN}$ model, these mice were resistant to collageninduced arthritis. These data indicated that the IL-23/Th17 axis is dispensable during the autoantibody-mediated effector phase of arthritis, whereas it is crucially involved in mounting an autoimmune response during CIA. Despite being protected from CIA, IL23 $\%$ mice displayed regular levels of anti-collagen antibodies, which also showed a regular avidity. Likewise, we observed no difference in the IgG subclasses between the two genotypes. Analysis of the glycosylation pattern of antibodies in the sera of WT and IL $23^{-\%}$ mice, however, revealed major differences in the content of sialic-acid and fucose residues at the Fc part of the IgGs resulting in an antiinflammatory IgG profile in the sera of IL23 $3^{--}$mice. The changes in the IgG glycosylation, in turn, correlated with changes in the expression pattern of glycosyltransferases in plasmablasts and plasmacells of WT and IL23-deficient mice.

Conclusions Together, these data show that the IL23/Th17 axis controls the degree of antibody glycosylation and, in turn, indicate that this regulation of the glycosylation of autoantibodies is a critical step in the pathogenesis of Th17-mediated autoimmune diseases such as RA.

\section{T cells - activation and regulation}

\section{A3.1 1.25(OH $)_{2} \mathrm{D}_{3}$ INHIBITS TH17 POLARISATION AND ROR $\gamma \mathrm{t}$ EXPRESSION THROUGH GATA3-DEPENDENT AND -INDEPENDENT MECHANISMS}

doi:10.1136/annrheumdis-2013-203216.1

1,2W Dankers, 1,2JP van Hamburg, 1,2 AMC Mus, 1,2PS Asmawidjaja, 1,20BJ Corneth, 1,2F Luk, 3.JPTM van Leeuwen, "RW Hendriks, ${ }^{5} \mathrm{~L}$ Boon, 1,6 EM Colin, 1,2E Lubberts. ${ }^{1}$ Departments of Rheumatology; ${ }^{2}$ Immunology; ${ }^{3}$ Internal Medicine; ${ }^{4}$ Pulmonary Medicine, Erasmus MC University Medical Center, Rotterdam, The Netherlands; ${ }^{5}$ Bioceros, Utrecht, The Netherlands; ${ }^{6}$ Department of Rheumatology, ZGT, Almelo, The Netherlands

Background and Objectives Vitamin D has suppressive effects on autoimmune diseases, such as rheumatoid arthritis (RA). Regulation of Th17 cell activity is an important mechanism by which vitamin $\mathrm{D}$ exerts these effects. Aside from inhibiting Th17 cytokines and the Th17 transcription factor ROR $\gamma$ t, vitamin D induces IL-4 and GATA3. Since GATA3 over-expression inhibits experimental Th17-mediated autoimmunity, we studied the contribution of GATA3 in vitamin D-mediated suppression of Th17 polarisation.

Methods Therefore $\mathrm{CD}^{+} \mathrm{T}$ cells were sorted from patients with early RA, naïve DBA-1 mice, DBA-1 mice immunised with collagen type II (CII) or naïve CD2-GATA3 transgenic mice and cultured under $\mathrm{T}$ helper cell polarising conditions with or without $1.25(\mathrm{OH})_{2} \mathrm{D}_{3}$, the active form of vitamin $\mathrm{D}$.

Results $1.25(\mathrm{OH})_{2} \mathrm{D}_{3}$ inhibits Th17 polarisation in CD4+ cells from both non-immunised and CII-immunised mice, while upregulating IL-4 and GATA3 expression. In these cultures, IL-4 inhibition partly reversed the vitamin D-mediated inhibition of Th17 polarisation. Moreover, GATA3 over-expression reduces Th17 differentiation to a lower level than $1.25(\mathrm{OH})_{2} \mathrm{D}_{3}$. Interestingly, combining GATA3 over-expression and $1.25(\mathrm{OH})_{2} \mathrm{D}_{3}$ treatment reduced IL-17A and ROR $\gamma t$ expression even further. Furthermore, geneexpression analysis showed that NFAT-C2, which is involved in IL$17 \mathrm{~A}$ production, was down-regulated by $1.25(\mathrm{OH})_{2} \mathrm{D}_{3}$. In addition, in T cells from patients with RA, $1.25(\mathrm{OH})_{2} \mathrm{D}_{3}$ inhibited Th17 cytokine and ROR $\gamma t$ expression and induced IL-4 and GATA3 expression. Conclusions These data show that vitamin $\mathrm{D}$-mediated regulation of Th17 polarisation occurs through GATA3-dependent mechanisms, including direct effects on ROR $\gamma \mathrm{t}$ expression and IL-4-mediated inhibition of Th17 polarisation. Moreover, GATA3-independent mechanisms are involved that may include modulation of NFAT-C2 expression.

\section{A3.2 A CD4 + T-CELL GENE EXPRESSION SIGNATURE PREDICTS DRUG SURVIVAL ON METHOTREXATE MONOTHERAPY IN EARLY RHEUMATOID ARTHRITIS}

doi:10.1136/annrheumdis-2013-203216.2

${ }^{1,2} \mathrm{AG}$ Pratt, $1 \mathrm{PM}$ Brown, ${ }^{3} \mathrm{SJ}$ Cockell, ${ }^{4} \mathrm{G}$ Wilson, 1,2JD Isaacs. ${ }^{1 / n s t i t u t e ~ o f ~ C e l l u l a r ~}$ Medicine (Musculoskeletal Research Group), Newcastle University, UK; ${ }^{2}$ Bioinformatics Support Unit, Newcastle University, UK; ${ }^{3}$ Musculoskeletal Unit, The Freeman Hospital, Newcastle-Upon-Tyne, UK; ${ }^{4}$ Faculty of Health and Social Care, University of Hull, UK

Background/Purpose The mechanism of action of methotrexate (MTX) in the management of rheumatoid arthritis (RA) remains 